\title{
UV-IR mixing and the quantum consistency of noncommutative gauge theories
}

\author{
Eric Nicholson ${ }^{\text {f }}$ \\ Theory Group, Department of Physics \\ University of Texas at Austin \\ Austin, TX 78712, USA
}

(Dated: November 14, 2018)

\begin{abstract}
We study the quantum mechanical consistency of noncommutative gauge theories by perturbatively analyzing the Wilsonian quantum effective action in the matrix formulation. In the process of integrating out UV states, we find new divergences having dual UV-IR interpretations and no analogues in ordinary quantum field theories. The appearance of these new UV-IR divergences has profound consequences for the renormalizability of the theory. In particular, renormalizability fails in any nonsupersymmetric noncommutative gauge theory. In fact, we argue that renormalizability generally fails in any noncommutative theory that allows quantum corrections beyond one-loop. Thus, it seems that noncommutative quantum theories are extremely sensitive to the UV, and only the softest UV behavior can be tolerated.

PACS numbers: 00

Keywords: Noncommutative Geometry, Matrix Theory, Dipole, UV-IR Mixing
\end{abstract}

\section{INTRODUCTION}

By now, it has been a few years since it was realized that noncommutative gauge theories emerge from string theory through various decoupling limits [1, 2]. Nonetheless, the understanding of the dynamics of noncommutative gauge theories remains in a relatively primitive state compared to ordinary gauge theories. The main difficulty is that noncommutative quantum field theories are highly nonlocal. In fact, one can think of the nonlocality as arising from elementary dipole degrees of freedom whose transverse length is proportional to their center of mass momentum [2]. The novel behavior of these dipole quanta gives rise to UV-IR mixing: UV dipoles grow long in spatial extent and mediate instantaneous long distance interactions that dominate in the IR.

Not surprisingly, UV-IR mixing leads to difficulties in the naive application of conventional field theory techniques to noncommutative theories. In particular, the non-decoupling of UV and IR states leads to ambiguities in the distinction between short and long distance physics. Consequently, there has been some confusion regarding such things as the renormalization of UV divergences [3, 4, 5, 6], the treatment of IR divergences [3, 7, 8], and Wilsonian integration [9, 10, 11]. What is more, the noncommutative gauge invariance is not preserved separately in each diagram of the standard perturbative expansion; rather, gauge invariance is achieved by an infinite resummation of diagrams [12, 13, 14]. Finally, there is a sort of naturalness problem with the conventional approach in that the intrinsic dipole structure of the elementary field quanta is not completely clear, although some suggestive results have been obtained [12].

On the other hand, in the matrix formulation of non-

*Electronic address: ean@physics.utexas.edu commutative gauge theory, the noncommutative gauge invariance is manifest [15], as is the dipole character of the elementary quanta [16]. In fact, as shown in [16], the matrix approach allows for a clear separation between the quantum effects of UV and IR dipoles. In particular, it was shown that one could make sense of Wilsonian integration despite UV-IR mixing. For example, the interactions that result from integrating out the UV states were explicitly calculated at both the one and two loop order. The resulting interactions were found to dominate the long distance behavior, which shed some light on UV-IR mixing in noncommutative gauge theory, as well as the nonanalytic dependence of the quantum theory on the noncommutativity parameter, $\theta$. For a different point of view on how the matrix formulation naturally leads to a bi-local representation, see 17.

However, while some progress has been made, there are still many unanswered questions. For one thing, the analysis of 16 was limited to perturbation theory, which generically breaks down due to strong quantum corrections. Although it was conjectured in [16] that the perturbative analysis of the Wilsonian quantum effective action is valid for supersymmetric theories whose UV behavior is softer, this remains to be proven. Furthermore, it is not even clear if noncommutative quantum theories are renormalizable, although there have been arguments for the affirmative 6 .

In this work, we continue to develop noncommutative gauge theory in the matrix formulation. In section II, after reviewing the basic ingredients of the dipole interpretation and the matrix formulation of noncommutative gauge theory, we determine the general form of the gauge invariant perturbative corrections to the quantum effective action that result from integrating out UV states. Based on this structure, we then show that the leading long distance interactions cancel at each order in perturbation theory for supersymmetric theories. In section III, we first discuss renormalizability in the context of nonsupersymmetric noncommutative theories and ar- 
gue that, beyond one loop order, UV-IR mixing generally introduces divergences that spoil the consistency of the theory. We are led to conjecture that only supersymmetric noncommutative theories that do not get renormalized beyond one loop can be consistent quantum mechanically. We end with some discussion of our results and some concluding remarks. The appendices contain a review of the important results of [16] and some other technical details.

\section{GAUGE INVARIANT STRUCTURE OF PERTURBATION THEORY}

Ultimately, we would like to further develop our intuition for how UV-IR mixing affects the quantum mechanical consistency of noncommutative gauge theories. To this end, it will prove convenient to separate the degrees of freedom into an UV region, defined by momenta much greater than any other scale in the problem, and an IR region defined by all of the rest of momenta. The strategy will be to first integrate out the UV modes in the Wilsonian sense, and then to study the effective action in the IR regime. In this section, we will discuss the general structure of perturbative corrections to the effective action resulting from integrating out UV states.

As discussed in 14, 18, the structure of perturbative corrections is strongly constrained by noncommutative gauge invariance, which is a much larger symmetry group than ordinary gauge symmetries. In fact, gauge invariant quantities receive contributions from an infinite number of diagrams in terms of the conventional perturbative expansion. For this reason, it is most convenient to work in the matrix formulation of noncommutative gauge theory, which is manifestly gauge invariant. The technical machinery useful for performing perturbative calculations in the matrix approach was developed in [16, although the main technical results from the matrix formulation, which will be used extensively in what is to follow, are briefly reviewed in Appendix A.

We will begin by recalling the interpretation of the matrix degrees of freedom in terms of dipoles, since this viewpoint is robust and will clearly generalize to all orders of perturbation theory. The dipole interpretation follows naturally from the representation of the matrix degrees of freedom in terms of the noncommutative gauge group. As discussed in [19], the degrees of freedom of noncommutative gauge theory are represented as infinite dimensional Hermitian matrices

$$
X^{i}(t)=\hat{x}^{i} \otimes \mathbb{1}_{N \times N}+\theta^{i j} A_{j}(\hat{x}, t)
$$

where $\hat{x}^{i}$ are time-independent Hermitian matrices satisfying the algebra of the noncommuting $2 p$-plane

$$
\left[\hat{x}^{i}, \hat{x}^{j}\right]=i \theta^{i j} \mathbb{1}
$$

The essential point is that $X^{i}$ lives in the adjoint representation of the group noncommutative $U(N)$ which, as indicated by the tensor product structure of Eq. (11), can be thought of as the product of ordinary groups $U(\infty) \otimes U(N)$. The representations of the ordinary $U(N)$ group will play the role of Chan-Paton factors joined to the ends of the dipoles, and their treatment will come naturally in our framework. The crucial observation is that the adjoint representation of $U(\infty)$, furnished by functions of $\hat{x}^{i}$, should be thought of in 't Hooft double line notation. In this language, the Feynman diagrams that appear in the matrix formulation have a simple and intuitive interpretation in terms of dipole degrees of freedom: the double lines literally represent the spatial trajectory that is traced out by the endpoints of the dipole quanta as they propagate around the loops [16].

In order to demonstrate and reinforce this dipole picture, we shall review the results of explicit calculations and their interpretations [16]. For example, consider the one loop diagram

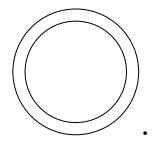

A simple matrix calculation gives an interaction term in the effective Lagrangian proportional to

$$
\begin{aligned}
\left(N_{B}-N_{F}\right) \int & d^{2 p} x_{1} d^{2 p} x_{2} \rho\left(x_{1}, t\right) \rho\left(x_{2}, t\right) \\
& \times \int \frac{d \omega}{2 \pi} \log \widetilde{G}\left(\omega, \theta^{-1} x_{12}\right),
\end{aligned}
$$

where $N_{B}$ and $N_{F}$ are the numbers of bosonic and fermionic polarization states, respectively, and the rest of the notation is reviewed in Appendix A. Note that although we have discussed only gauge field degrees of freedom explicitly, fermions and other matter fields will generally contribute quantities of the same form as the pure gauge field calculation, but they will differ in the constant of proportionality as in Eq. (3) above.

The $\log \widetilde{G}\left(\omega, \theta^{-1} x_{12}\right)$ factor that appears in the integrand of Eq. (3) is familiar from field theory if we identify the momentum of the virtual quantum with $p_{i}=\theta_{i j}^{-1}\left(x_{1}^{j}-x_{2}^{j}\right)$. In fact, this is precisely the relation between the center of mass momentum and the end points of the dipoles that is expected [2]! The most obvious consequence of this relation is that $\mathrm{UV}$ dipoles are long in spatial extent. Therefore, if we are to associate the double lines of the matrix diagrams with the ends of the dipoles, we should think of the one loop diagram above "stretched out" into a long thin cylinder as depicted in FIG. 1.

The quantity $\rho(x, t)$ which appears in Eq. (3) is a gauge invariant functional of the low momentum background field that is well localized in space around $x$, given the fact that we are only integrating out high momentum states, as discussed in [16]. Evidently, we are to associate a trace, which produces the gauge invariant functional $\rho$, to each boundary of the cylinder. In fact, this leads to the connection between Eq. (3) and the field theory diagrams 


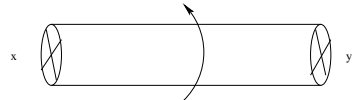

FIG. 1: High momentum virtual dipoles grow long in the transverse direction and mediate instantaneous interactions between distant background fluctuations at $x_{1}$ and $x_{2}$.

that appear in the conventional perturbative expansion. If we expand $\rho(x)=\operatorname{tr}_{N}(\mathbb{1})+\Delta(x)$, then as discussed in [16], we identify the constant term as the contribution from field theory diagrams with no background field insertions on the corresponding boundary, while the term with nontrivial position dependence descends from diagrams that include background gauge field insertions on the corresponding boundary. This interpretation is supported by the fact that $\Delta(x)=0$ for field configurations gauge equivalent to $A_{i}(x)=0$. Using this reasoning, we can conclude that the vacuum diagram, which involves no insertions on either boundary, is proportional to

$$
\left(N_{B}-N_{F}\right) N^{2} V \int \frac{d \omega d^{2 p} p}{2 \pi(2 \pi)^{2 p}} \log \widetilde{G}(\omega, p) .
$$

Of course, this is just the expected result from field theory.

The contribution from diagrams with planar background field insertions vanishes because $\int d^{2 p} x \Delta(x, t)=$ 0 ; however, the nonplanar diagrams yield a nontrivial interaction term proportional to

$$
\begin{aligned}
\left(N_{B}-N_{F}\right) \int & d^{2 p} x_{1} d^{2 p} x_{2} \Delta\left(x_{1}, t\right) \Delta\left(x_{2}, t\right) \\
& \times \int \frac{d \omega}{2 \pi} \log \widetilde{G}\left(\omega, \theta^{-1} x_{12}\right) .
\end{aligned}
$$

Apparently, Eq. (5) describes the instantaneous interaction between distant background fluctuations at $x_{1}$ and $x_{2}$, as illustrated in FIG. 11. In fact, this interaction term demonstrates the novel feature of noncommutative quantum field theories: UV dipoles grow long in spatial extent and mediate instantaneous long distance interactions, which is how we can think about UV-IR mixing.

However, it should be noted that, in the case of Eq. (5), the interactions grow strong at long distances because

$$
\int \frac{d \omega}{2 \pi} \log \widetilde{G}\left(\omega, \theta^{-1} x_{12}\right) \sim\left|x_{1}-x_{2}\right|+\text { const. }
$$

As discussed in 15 , the strong interaction expressed in Eq. (6) is due to the leading IR poles in external momenta that result from the UV region of loop integrals involving nonplanar background insertions. The appearance of nonanalytic behavior in external momenta has also been discussed from the field theory perspective in [20]. In the particular case of Eq. (6), we will require that $N_{B}=N_{F}$ so that this term vanishes and perturbation theory remains valid. However, the appearance of strong IR corrections from the quantum effects of virtual UV states and their treatment in general will be a central theme of this work.

Furthermore, as discussed in [16], it is important to realize that the one loop matrix calculation (3) gives only the leading term in a derivative expansion involving the background field. The next to leading order one loop diagram involves two extra insertions of the background field strength

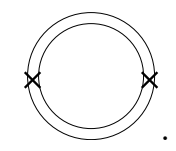

The contribution from this diagram was shown to be of the form

$$
\begin{array}{r}
\int d^{2 p} x_{1} d^{2 p} x_{2}\left[c_{1} \rho_{F F}\left(x_{1}, t\right) \rho\left(x_{2}, t\right)+c_{2} \rho_{F}\left(x_{1}, t\right)\right. \\
\left.\times \rho_{F}\left(x_{2}, t\right)\right] \int \frac{d \omega}{2 \pi} \widetilde{G}\left(\omega, \theta^{-1} x_{12}\right) \widetilde{G}\left(\omega, \theta^{-1} x_{21}\right),
\end{array}
$$

where $c_{1}$ and $c_{2}$ are constants depending on the dimensionality and the matter content of the theory, as previously discussed. Notice that a nontrivial contribution from planar field theory diagrams is contained in the first term above.

We will discuss Eq. (7) in much more detail in the next section, but for now, we shall just remark on its basic features. Evidently, the field strength operators that were inserted into the matrix diagram above appear as operators attached to the end of Wilson lines. This structure seems to reinforce the correspondence between the boundaries of the matrix diagram and traces which lead to gauge invariant Wilson lines. In general, higher order terms in the derivative expansion involve higher dimensional operator insertions into the loop diagram, and hence, the Wilson lines. However, the double trace structure and the corresponding interpretation in terms of long distance two-body interactions remains the same. Moreover, it is interesting that the appearance of the propagator $\widetilde{G}$ in Eq. (7) is again consistent with our field theory intuition.

Higher loop diagrams, on the other hand, will involve more traces, and will therefore lead to multi-body interactions. For example the leading two-loop contribution to the effective Lagrangian comes from

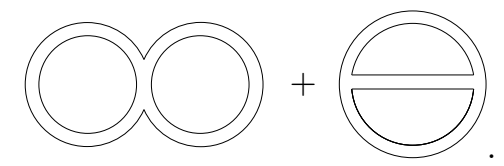

Note that we have not included the nonplanar matrix diagrams because their contribution to the Wilsonian integration is exponentially suppressed due to Moyal phase factors [16]. As indicated by the double line diagrams above, the leading two-loop diagrams give triple trace contributions. The first diagram gives an interaction term in the effective Lagrangian proportional to [16]

$$
\int d^{2 p} x_{1} d^{2 p} x_{2} d^{2 p} x_{3} \rho\left(x_{1}, t\right) \rho\left(x_{2}, t\right) \rho\left(x_{3}, t\right) \int \frac{d \omega_{1}}{2 \pi} \frac{d \omega_{2}}{2 \pi}
$$




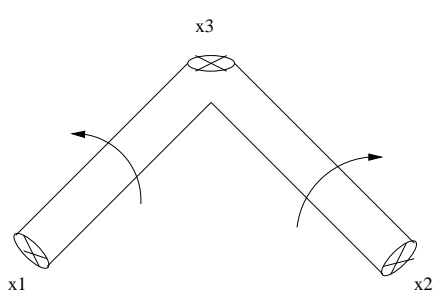

(a) An illustration of the contribution from the firstorder treatment of quartic interaction terms given by (\$)

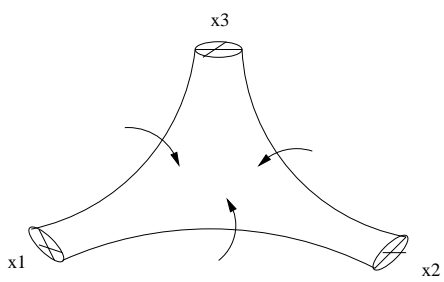

(b) An illustration of the contribution from the second order treatment of cubic interaction terms given by (9).

FIG. 2: Long distance three-body interactions corresponding to high momentum dipoles propagating in two-loop diagrams.

$$
\times \widetilde{G}\left(\omega_{1}, \theta^{-1} x_{13}\right) \widetilde{G}\left(\omega_{2}, \theta^{-1} x_{23}\right)
$$

and, as calculated in Appendix B, the second gives a term proportional to

$$
\begin{aligned}
\int d^{2 p} & x_{1} d^{2 p} x_{2} d^{2 p} x_{3} \rho\left(x_{1}, t\right) \rho\left(x_{2}, t\right) \rho\left(x_{3}, t\right) \int \frac{d \omega_{1}}{2 \pi} \frac{d \omega_{2}}{2 \pi} \\
& \times\left(\omega_{1} \omega_{2}-x_{12} \cdot x_{23}\right) \widetilde{G}\left(\omega_{1}, \theta^{-1} x_{12}\right) \\
& \times \widetilde{G}\left(\omega_{2}, \theta^{-1} x_{23}\right) \widetilde{G}\left(\omega_{1}+\omega_{2}, \theta^{-1} x_{31}\right) .
\end{aligned}
$$

Indeed, as expected based on our intuition, Eqs. (8) and (9) describe long distance interactions that arise from high momentum dipoles growing large in spatial extent and "stretching out" the matrix diagrams, as depicted in FIG. 2. Furthermore, these expressions bear a close resemblance to what is expected from ordinary field theory, and in fact, the general structure of perturbative corrections is starting to emerge.

To each boundary of the double line diagram we should associate a point in space and a trace which yields a gauge invariant Wilson line. The position dependence of the interaction strength between the Wilson lines follows from an integral over frequencies with the integrand being given by a particular function of both the dipole frequencies and momenta as defined by the separation between the space points associated to the boundaries of the double line diagram. In fact, the particular function of dipole frequencies and momenta corresponds precisely to the structure of momentum space field theory propagators that appear in the analogous process in ordinary field theory.
The subleading terms in the derivative expansion will have a similar trace structure but Wilson lines modified to include higher derivative operator insertions as in Eq. (7). Naturally, the subleading terms will include a different function of dipole frequencies and momenta that reflects the extra propagators that are required by the operator insertions. Note that for dipole degrees of freedom, powers of momentum are equivalent to powers of separation, as discussed in [16] and in Appendix A. Therefore, the inclusion of more powers of momentum in the denominator leads to faster falloff with distance, which is expected from subleading terms in a derivative expansion. However, quantum corrections coming from higher loop orders are generally strong, as we will discuss in the next section.

Of more importance to our immediate goal, is the determination of the form of the leading long distance interactions at each order in perturbation theory. It is clear from the general structure discussed above that at $L$-loop order the leading long distance interactions will involve $L+1$ Wilson lines with no operator insertions, such as Eq. (3) in the case $L=1$ and both Eq. (8) and Eq. (9) in the case $L=2$. The crucial observation is that since the vacuum diagrams are contained in interactions of this form, as shown explicitly by Eq. (4) in the one-loop case, these terms must vanish entirely if the vacuum diagrams vanish. Thus, in supersymmetric noncommutative theories, the leading long distance interactions must cancel at each order in perturbation theory.

This statement is a generalization to all loop orders of the cancellation that we have discussed in the case of the leading one-loop interactions given by Eq. (3). It reflects the fact that supersymmetric theories are softer in the UV, and hence, the IR behavior that is generated by UVIR mixing is not as strong. In fact, the cancellation of the leading IR interactions occurs even in minimally supersymmetric theories. The relation between the amount of supersymmetry and the cancellation of other terms has been discussed in [8, 9, 20, 21] from the field theory perspective, and we will touch on this important point as well in the next section.

In summary, we have determined the gauge invariant form of perturbative corrections to the Wilsonian quantum effective action for noncommutative gauge theories. It followed immediately from the structure of perturbation theory that the leading long distance interactions cancel order by order in the supersymmetric theories. Since nonsupersymmetric theories do not enjoy these cancellations, they are strongly coupled at long distances. In fact, what we are beginning to discover is that noncommutative quantum theories are highly sensitive to the UV, which we will further explore in the next section.

\section{RENORMALIZABILITY}

In the previous section, we determined the gauge invariant form of the perturbative quantum corrections to 
the effective action that result from integrating out UV states. In this section we will take a closer look at these perturbative corrections and their divergence properties. In addition to the expected types of UV divergences, we will find new divergences that have dual UV and IR interpretations and for which there are no analogues in ordinary quantum field theories. In fact, we will argue that these new UV-IR divergences, if they are allowed, will spoil the renormalizability of the theory. In the end, we will find that only supersymmetric theories with the softest UV behavior can be consistent quantum mechanically.

Because it will set the ground work for the discussion of the more interesting supersymmetric case, we will start this section by discussing divergences in the context of nonsupersymmetric noncommutative gauge theories. We will begin with the familiar procedure of gauge coupling renormalization. In fact, in this case, it will turn out that renormalizability is threatened already at the two loop level. To see how this happens, we first recall from [16] the form of the one-loop quantum correction to the gauge coupling. The only candidate for this type of term is Eq. (7) because it comes from a one-loop graph with two insertions of the field strength operator. To isolate the UV divergence in the planar diagrams, we must use the splitting scheme $\rho(x)=\operatorname{tr}_{N}(\mathbb{1})+\Delta(x)$ and focus on the first term, as previously discussed. The integral then factorizes into

$$
\begin{aligned}
& c_{1} \int d^{2 p} x_{1} \rho_{F F}\left(x_{1}, t\right) N \int \frac{d \omega}{2 \pi} d^{2 p} x_{12} \widetilde{G}\left(\omega, \theta^{-1} x_{12}\right)^{2} \\
= & c_{1} \operatorname{Tr}\left(\left[B^{i}, B^{j}\right]^{2}\right) N \int \frac{d \omega d^{2 p} p}{2 \pi(2 \pi)^{2 p}} \widetilde{G}(\omega, p)^{2} .
\end{aligned}
$$

This quantity is easily recognized as contributing to the renormalization of the operator $\operatorname{Tr}\left[B^{i}, B^{j}\right]^{2}$, as expected. Therefore, the one-loop quantum correction to the gauge coupling is proportional to the dimensionless quantity

$$
g^{2} N \int \frac{d \omega d^{2 p} p}{2 \pi(2 \pi)^{2 p}} \widetilde{G}(\omega, p)^{2},
$$

where we have restored the gauge coupling $g^{2}$ explicitly.

If we are to consistently absorb the one-loop correction (11) into a new quantum corrected gauge coupling, we must find the same quantity at higher loops correcting each factor of $g^{2}$ that appears. Otherwise, renormalizability will be threatened, because we will be forced to introduce new couplings into the effective Lagrangian in order to cancel all of the resulting divergences. However, in our conventions, the dependence on the gauge coupling is particularly simple - powers of $g^{2}$ count the number of loops. Thus, powers of the gauge coupling only appear multiplying the loop corrections; the Wilson lines, which encode the background gauge field insertions into the loops, are independent of the coupling. Therefore, to test renormalizability of the gauge coupling at the two loop order, we should look for a term of the form

$$
\int d^{2 p} x_{1} d^{2 p} x_{2} \rho\left(x_{1}, t\right) \rho\left(x_{2}, t\right)\left|x_{1}-x_{2}\right|
$$

$$
\times \int \frac{d \omega d^{2 p} p}{2 \pi(2 \pi)^{2 p}} \widetilde{G}(\omega, p)^{2},
$$

because it corresponds to the one-loop correction to $g^{2}$ given by Eq. (11) multiplying the leading one-loop interaction (3).

In order to find this sort of term, we consider the leading two-loop interactions. As discussed in Appendix B, these contributions can be combined into the form of Eq. (8). It is now straight forward to isolate the divergent quantum corrections to the two-body interactions. The constant term in either $\rho\left(x_{1}\right)$ or $\rho\left(x_{2}\right)$ gives

$$
\begin{aligned}
& \int d^{2 p} x_{1} d^{2 p} x_{3} \rho\left(x_{1}, t\right) \rho\left(x_{3}, t\right) \int \frac{d \omega_{1}}{2 \pi} \widetilde{G}\left(\omega_{1}, \theta^{-1} x_{13}\right) \\
& \quad \times \int \frac{d \omega_{2} d^{2 p} p_{2}}{2 \pi(2 \pi)^{2 p}} \widetilde{G}\left(\omega_{2}, p_{2}\right) .
\end{aligned}
$$

Apparently, Eq. (13) involves the leading one-loop UV divergent contribution to the mass term of the field theory propagator $\widetilde{G}$ appearing in Eq. (3). In fact, this type of correction is familiar from ordinary quantum field theories. However, it will turn out that in the supersymmetric theories of ultimate interest to us, corrections of this form will not appear. In any case, Eq. (13) is not the term we are looking for, so we move on.

The only other candidate for the appearance of Eq. (12) in the leading order two-loop diagrams is the interaction that comes from the constant term of $\rho\left(x_{3}\right)$

$$
\begin{aligned}
& \int d^{2 p} x_{1} d^{2 p} x_{2} \rho\left(x_{1}, t\right) \rho\left(x_{2}, t\right) \int \frac{d \omega_{1}}{2 \pi} \\
& \quad \times \int \frac{d \omega_{2} d^{2 p} p_{3}}{2 \pi(2 \pi)^{2 p}} \widetilde{G}\left(\omega_{21}, p_{3}-\theta^{-1} x_{12}\right) \widetilde{G}\left(\omega_{2}, p_{3}\right) .
\end{aligned}
$$

Needless to say, Eq. (14) is not the term we need either; thus, the renormalizability of the gauge coupling is threatened already at the two-loop order. However, a closer look at Eq. (14) reveals an entirely new problem facing the renormalizability of noncommutative theories that we will now discuss.

Evidently, Eq. (14) represents a two-loop quantum correction to the leading one-loop two-body interaction (3). The growth in $\left|x_{1}-x_{2}\right|$ of the interaction strength of Eq. (14) is given by

$$
\int \frac{d \omega_{1}}{2 \pi} \frac{d \omega_{2} d^{2 p} p_{3}}{2 \pi(2 \pi)^{2 p}} \widetilde{G}\left(\omega_{21}, p_{3}-\theta^{-1} x_{12}\right) \widetilde{G}\left(\omega_{2}, p_{3}\right) .
$$

Clearly, Eq. (15) is UV divergent for $p \geq 1$, so we must introduce an UV cutoff $M$. The integral can now be calculated in a straight forward fashion and the leading dependence on separation goes as $\left|x_{1}-x_{2}\right|^{2 p-2} \log \left(\mid x_{1}-\right.$ $\left.\left.x_{2}\right|^{2} / M^{2}\right)$. However, since there is no two-body interaction that grows as $\left|x_{1}-x_{2}\right|^{2 p-2}$ at one loop, this logarithmic divergence cannot be absorbed into the gauge coupling. Therefore, a new coupling for a two-body interaction of the form

$$
\int d^{2 p} x_{1} d^{2 p} x_{2} \rho\left(x_{1}\right) \rho\left(x_{2}\right)\left|x_{1}-x_{2}\right|^{2 p-2}
$$


must be introduced into the effective Lagrangian so that the divergence in Eq. 14 can get cancelled.

Although one can arrange for the cancellation of divergent corrections to any interaction strength by continuing to introduce new interactions with divergent couplings, as above, it is not hard to see that this procedure is a serious threat to the renormalizability of the theory. To see this, we need only note that, at higher orders, there will be quantum corrections to the interaction strengths that come in the form of powers of the dimensionless quantity

$$
\frac{g^{2}}{\left|x-x^{\prime}\right|^{3-2 p}} \log \left(\left|x-x^{\prime}\right|^{2} / M^{2}\right) \text {. }
$$

Therefore, to cancel all of the divergences that appear in the various powers of (17), we would have to introduce an infinite number of new interactions. The reason is that there are an infinite number of distinct functions of $\left|x-x^{\prime}\right|$ that can appear, each requiring a different interaction term with its own coupling in order to cancel the required divergences in the effective Lagrangian. Thus, it seems that divergent corrections to the interaction strength between Wilson lines, a new feature of noncommutative theories having no analogue in ordinary quantum field theories, will generally spoil the renormalizability of the theory.

A crucial assumption in the argument above is that if divergent quantum corrections to interaction strengths occur at the two-loop order, they will appear at all higher orders as well. In fact, this is easy to see graphically by recalling the relation between matrix diagrams and field theory diagrams. Using the correspondence that we discussed earlier in section II, we find that Eq. (14) descends from the leading order terms in the external momenta expansion of field theory diagrams of the form

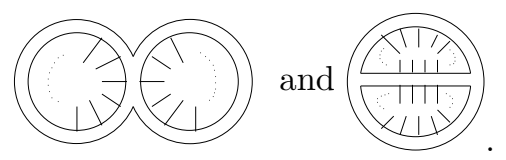

Evidently, the dangerous divergences are not due to planar subdiagrams, which have the standard UV interpretation, but are due to purely nonplanar field theory diagrams. We will develop more understanding for what this means in the moment, but for now let us note that higher order graphs of this type exist at each loop order in perturbation theory. For example, FIG. 3 depicts some three-loop field theory diagrams that give rise to divergent quantum corrections to three-body and two-body interactions. Thus, dangerous corrections to interaction strengths that first appear at some given order in perturbation theory will receive quantum corrections from all higher loop orders, generating corrections in the form of powers of (17).

However, by the arguments above, the divergences appearing in powers of Eq. (17) will inevitably spoil renormalizability. Therefore, any renormalizable noncommutative theory cannot allow the leading order terms in the derivative expansion, which as we have seen, contain

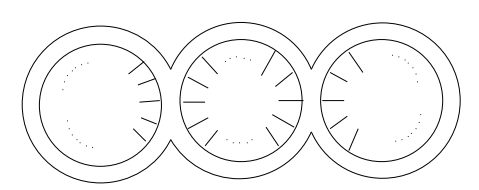

(a) Some three-loop field theory diagrams containing dangerous quantum corrections to the two-loop three-body interactions.

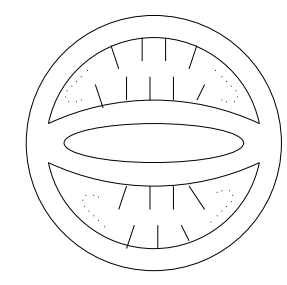

(b) Some three-loop field theory diagrams containing dangerous quantum corrections to the one-loop two-body interactions.

FIG. 3: Examples of three-loop nonplanar field theory diagrams that contribute dangerous quantum corrections to the strength of lower order interactions.

these dangerous corrections. We are forced to conclude that only supersymmetric noncommutative theories can be renormalizable since, in this case, the leading order interactions always vanish.

Before discussing the supersymmetric theories, let us take some time to understand the nature of the dangerous divergences by recasting them into the form of momentum integrals. For example, consider the transformation of Eq. (14) to momentum space

$$
\begin{gathered}
\int \frac{d^{2 p} k}{(2 \pi)^{2 p}} \widetilde{\rho}(k, t) \widetilde{\rho}(-k, t) \frac{d \omega_{1} d^{2 p} q}{2 \pi(2 \pi)^{2 p}} \frac{d \omega_{2} d^{2 p} p}{2 \pi(2 \pi)^{2 p}} \\
\quad \times e^{i k \cdot \theta \cdot q} \widetilde{G}\left(\omega_{21}, p-q\right) \widetilde{G}\left(\omega_{2}, p\right) .
\end{gathered}
$$

We can first perform the integrals over $\omega_{2}$ and $p$ by introducing a Schwinger parameter and an UV cutoff $M$, as in 3. 3. Then the integral over $\omega_{1}$ and $q$ is regular, and we are left with a quantity proportional to

$$
\begin{gathered}
\int \frac{d^{2 p} k}{(2 \pi)^{2 p}} \frac{\widetilde{\rho}(k, t) \widetilde{\rho}(-k, t)}{\left(k^{2}+1 / M^{2}\right)^{2 p-1}} \\
=\int d^{2 p} x_{1} d^{2 p} x_{2} \rho\left(x_{1}, t\right) \rho\left(x_{2}, t\right) \\
\quad \times \int \frac{d^{2 p} k}{(2 \pi)^{2 p}} \frac{e^{i k \cdot\left(x_{1}-x_{2}\right)}}{\left(k^{2}+1 / M^{2}\right)^{2 p-1}} .
\end{gathered}
$$

Now the $M \rightarrow \infty$ limit results in an IR divergence instead of an UV divergence, and $1 / M$ plays the role of an IR cutoff! The transform back into position space just gives the same correction to the interaction strength that we have already determined directly from Eq. (15).

Apparently, these types of divergences have a dual UVIR interpretation. The origin of this duality can be traced 
to the Moyal phase factor $\exp (i k \cdot \theta \cdot q)$ in Eq. (18), which represents the nonplanarity of the background insertions and gives rise to UV-IR mixing. In particular, the UV loop integrals over virtual states, labelled by momenta $p$ and $q$, produce nonanalytic dependence on the background momentum $k$ that becomes important in the IR. Thus, on one hand, the divergence comes from integrating over UV states, but on the other hand, the divergence cast be recast into the form of an IR singular Fourier transform.

Actually, as discussed in [16], this type of UV-IR mixing has a simple interpretation in terms of dipole degrees of freedom: the separation between the endpoints of the dipoles is the dual variable to the external momenta $k$; therefore, the IR singular Fourier transform gives rise to corrections to interaction strengths which grow strong with large separation; but, since powers of separation are equivalent to powers of dipole momentum, these strong corrections can also be thought of as arising from UV divergences in the theory. Thus, the dipole intuition that emerges from the matrix formulation seems to shed new light on the proposal of [5, 21 concerning the dual interpretation of divergences due to nonplanar diagrams.

In fact, at this point, let us take an aside to remark on the virtues of the matrix formulation of noncommutative gauge theory. First of all, the matrix degrees of freedom naturally encode the elementary dipole structure of the field quanta, and hence, the essence of UV-IR mixing. This is a very convenient aspect of the matrix approach, which leads to great conceptual clarity in the physics of noncommutative quantum theories, as we have seen. Perhaps, the most important advantage of the matrix description, however, is the manifest nature of the noncommutative gauge invariance. This crucial property allows us to distinguish between various long distance interactions, which in turn, forms the basis for our interpretation of the UV-IR divergences that appear in the theory.

Back to the issue of divergences in supersymmetric noncommutative theories, we now consider interactions that are of next to leading order in the derivative expansion. As we have discussed, these are the lowest order terms that do not generally vanish in the case of supersymmetric theories. However, we will find that UV-IR divergences do generally occur in the next to leading order interactions, and therefore, supersymmetric theories generally suffer from UV-IR divergences, as well. For example, as derived in Appendix Q , the next to leading order two-loop interactions include dangerous terms such as Eq. (C3), which contain a quantum correction to the interaction strength of Eq. (7) that goes as

$$
\begin{aligned}
& \int d \omega_{1} d \omega_{2} d^{2 p} x_{3} \widetilde{G}\left(\omega_{1}, \theta^{-1} x_{12}\right)^{2} \widetilde{G}\left(\omega_{2}, \theta^{-1} x_{23}\right) \\
& \times \widetilde{G}\left(\omega_{1}+\omega_{2}, \theta^{-1} x_{13}\right) .
\end{aligned}
$$

Following an analysis parallel to the one above for Eq. (15), it is straight forward to show that UV-IR divergences appear in Eq. (20) for $p \geq 3 / 2$ (Note that by $p$ equal to a half-integer we really mean that, in addition to $p-1 / 2$ noncommutative two-planes, there is an additional commutative spatial direction. The proper way to include this case in our analysis is to let $p$ remain the integer valued number of noncommutative two-planes and replace $d \omega$ with $d^{2} \omega$ to represent the two commuting spacetime directions. All of the conclusions that we have drawn up to this point are true also in the case when the space dimensionality is odd).

Furthermore, the graphical argument that we employed earlier in the case of the leading order interactions, can be easily generalized to include the next to leading order terms in the derivative expansion. We need only consider adding higher derivative operator insertions in the matrix framework or, in the field theory picture as in FIG. 3, taking the next to leading order terms in the expansion in external momenta. In any case, we are forced to conclude that if dangerous UV-IR terms appear at two loops, then there will be more dangerous quantum corrections that emerge from all higher loop orders. Therefore, if Eq. (C3) appears in 3+1 dimensions or higher, UVIR divergences will proliferate and renormalizability will fail.

In order to determine what corrections are allowed by supersymmetric theories, let us take some time to discuss the proportionality constants that we have so far ignored. This will be fruitful because we will find that the nonrenormalization theorems enjoyed by ordinary supersymmetric theories have a natural extension to noncommutative theories. We can then use these nonrenormalization theorems to show that $3+1$ dimensional noncommutative theories with a sufficiently high degree of supersymmetry do not allow UV-IR divergences, and therefore, renormalizability does not face any obvious obstruction in these special theories.

We begin by considering the content of the matrix diagrams from a field theory point of view. First of all, the double line matrix diagrams themselves only contain information pertaining to the contraction of gauge indices, which encodes the planarity and nonplanarity of both the external operator insertions and the internal propagators. Thus, the double line diagrams contain purely topological information, not specific to any particular gauge theory - ordinary or noncommutative. In terms of the framework that we have discussed up to this point, this topological information only fixes the ratio of coefficients between terms with a similar propagator structure but different Wilson line structure. For example, $c_{1} / c_{2}$ is fixed by topological considerations, as are both $b_{6} / b_{7}$ and $b_{6} / b_{8}$. Furthermore, the coefficients coming from nonplanar matrix diagrams, which we have not discussed here, are fixed relative to the coefficients of the corresponding planar diagrams (Note that factors of $N$ are contained in the traces of the Wilson lines, not the coefficients that we are referring to). However, topological quantities, such as the ratio between coefficients, are independent of any characteristic specific to the theory in question such as the field content, the allowed couplings between fields, 
and whether the theory is noncommutative or not.

Although the ratios of certain coefficients are topological quantities, independent of the theory in question, the coefficients coming from planar and nonplanar matrix diagrams involving a similar propagator structure all share a common normalization factor that depends on the number and type of modes that propagate around the loops. Thus, the overall normalization factor does depend on characteristics specific to a particular theory, such as the field content, the allowed couplings between fields, and the coupling constants. However, the noncommutativity of the theory enters only through the Moyal phase factors inside the momentum integrals of nonplanar field theory diagrams; noncommutativity does not affect the coefficient outside the integral. It follows that a given coefficient in a particular noncommutative gauge theory is identical to the same coefficient in the corresponding ordinary gauge theory. After all, with the exception of Moyal phase factors in the the noncommutative theories, the contraction of fields is the same in both cases. Therefore, the nonrenormalization theorems of ordinary supersymmetric theories generalize immediately to noncommutative supersymmetric theories.

As an application of this useful result, let us return to the problem of dangerous UV-IR divergent terms in the next to leading order two-loop contribution to the quantum effective action, which are given by Eq. (C3). According to our reasoning above, $b_{6}, b_{7}$, and $b_{8}$ are all proportional to the two-loop contribution to the beta function. Therefore, we can guarantee their vanishing by imposing $\mathcal{N}=2$ supersymmetry in $3+1$ dimensions. In this case, the dangerous two-loop interactions cancel, as do all higher loop corrections. Moreover, the two-loop terms given by Eq. (C1) cancel because no mass terms are allowed to appear perturbatively, given any amount of supersymmetry. Thus, renormalizability for $3+1$ dimensional noncommutative gauge theories requires at least $\mathcal{N}=2$ supersymmetry, in which case the next to leading order interactions are restricted to the one-loop result given by Eq. (7), along with the two-loop terms containing the one-loop correction to the gauge coupling ( $\mathrm{C} 2)$. In the case of $\mathcal{N}=4$ supersymmetry, none of the next to leading order interactions are allowed what so ever.

Moreover, it is also worth mentioning that $\mathcal{N} \geq 2$ supersymmetry also implies that most, if not all, of the next to leading order nonplanar matrix diagrams cancel as well. The reason is that, as we have discussed, the coefficients of these contributions differ from their planar counterparts only by a combinatoric factor. Thus, the only next to leading order nonplanar remnant is

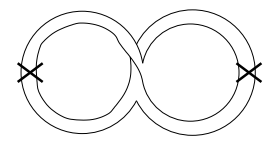

which is proportional to the one-loop beta function. Of course, all of the leading order nonplanar diagrams cancel, given any amount of supersymmetry. The advantage of eliminating the leading and next to leading order nonplanar matrix diagrams, is that they are divergent when the Wilsonian cutoff is removed. In fact, these divergences resulting from the IR region of integration in nonplanar loop integrals, have posed a major challenge to our understanding of the quantum effects of IR states in noncommutative theories. Therefore, it is fortunate that renormalizability of UV quantum fluctuations turns out to also constrain most of these IR divergences to vanish as well. Although a thorough treatment of the quantum effects of IR states is beyond the scope of this work, it seems that our results here are already a help in this direction.

While we have shown that $\mathcal{N} \geq 2$ supersymmetry is sufficient to prevent UV-IR divergences from appearing in diagrams containing two insertions of the field strength, we should still worry about dangerous terms appearing from diagrams involving other operator insertions. For example, all orders of wave function renormalization for matter hypermultiplets is generally allowed by $\mathcal{N}=2$ theories, although $\mathcal{N}=4$ theories do not allow any renormalization at all. Therefore, $\mathcal{N}=2$ gauge theories generally involve new UV divergences at each loop order, which will inevitably lead to the proliferation of dangerous UV-IR divergences via UV-IR mixing. After all, the divergent integral

$$
\int \frac{d \omega_{2} d^{2 p} p_{3}}{2 \pi(2 \pi)^{2 p}} \widetilde{G}\left(\omega_{2}, p_{3}\right) \widetilde{G}\left(\omega_{2}+\omega_{1}, p_{3}+\theta^{-1} x_{12}\right),
$$

that appears embedded in both Eqs. (15) and (20) will certainly appear at two loops and beyond in diagrams containing the insertion of any marginal operator, unless new divergences beyond one loop are not allowed. Thus, divergent quantum corrections beyond one loop are inherently dangerous in noncommutative theories.

We are led to conjecture that the only renormalizable, and hence, quantum mechanically consistent noncommutative gauge theories are supersymmetric and do not require renormalization beyond the one loop order. It is striking how strong this statement is. Apparently, noncommutative quantum theories are extremely sensitive to the UV, and only the softest UV behavior can be tolerated. In particular, the allowed $2+1$ dimensional theories include any supersymmetric gauge theory, and the allowed $3+1$ dimensional theories include both $\mathcal{N}=2$ and $\mathcal{N}=4$ noncommutative super Yang-Mills (NCSYM) theories plus some $\mathcal{N}=2$ gauge theories that include matter hypermultiplets in special representations.

It is interesting that $\mathcal{N}=1$ supersymmetry, or even $\mathcal{N}=2$ in most cases, does not appear to be enough to render $3+1$ dimensional noncommutative gauge theories renormalizable. Moreover, it is tempting to extend our conjecture to noncommutative theories other than gauge theories. After all, the intuition that we have gained appears to be quite generic and most likely applies to any noncommutative theory. For example, it seems unlikely that noncommutative scalar theory is consistent quantum mechanically, when nonsupersymmetric gauge 
theories are not. It even seems reasonable that renormalizability could fail in $\mathcal{N}=1$ and $\mathcal{N}=2$ supersymmetric noncommutative theories that do not include gauge degrees of freedom, in light of the fact that any extra hypermultiplets of matter that are added to $\mathcal{N}=2$ NCSYM theory must form special representations which do not allow wave function renormalization, so as not to spoil the theory.

Nonetheless, arguments have been put forward for the renormalizability of many noncommutative theories that are excluded by our conjecture [6]. Although, these works have involved different approaches which have encoded UV-IR mixing in one way or another, none have employed a manifestly dipole construction such as the matrix formulation. Since the dipole behavior of the elementary quanta is the fundamental origin of UV-IR mixing in noncommutative theories, it seems that the physical interpretation that naturally emerges from the matrix approach is the most reliable. We believe that this is a tremendous advantage when discussing renormalizability, because traditionally, the proper treatment of divergences has resulted from a sound physical interpretation for their meaning. Of course, the physical content of noncommutative theories is independent of the language used to discuss them; we are simply suggesting that the physics is more clear in the matrix representation. In any case, the difference between our work and [6] is the interpretation and treatment of the dual UV-IR divergences that occur in noncommutative quantum theories.

Before closing this section, let us make a few comments concerning the scope of our results from the perspective of string theory. Our analysis has been limited to noncommutative gauge theories, and given these dipole degrees of freedom, we have shown that both a sufficiently high degree of supersymmetry and low spacetime dimensionality is necessary to ensure the quantum mechanical consistency of the theory. However, this result does not imply that other degrees of freedom cannot be added to the theory to give a consistent UV completion. For example, 22] shows that there is an UV completion of some higher dimensional NCSYM theories in the form of noncommutative open string theories, in which the closed string sector has decoupled but there are still stringy modes from the open string sector that remain. Yet another distinct possibility is that, in the case of theories with a lesser degree of supersymmetry, there could be some closed string modes that survive the decoupling limit and render the theory consistent [23]. The point is that any noncommutative theory that emerges from a decoupling limit of string theory will be consistent; our results only imply that the decoupled theory can conceivably be a noncommutative gauge theory in only very special cases. String theory aside, however, the renormalizability of noncommutative quantum field theories is interesting in its own right.

\section{DISCUSSION AND OUTLOOK}

In this work, we have continued to develop noncommutative gauge theory in the matrix formulation. After reviewing the dipole interpretation of the matrix approach, we determined the general gauge invariant form of perturbative corrections to the quantum effective action that result from integrating out UV states. We then studied the divergence structure of these quantum corrections, which revealed new divergences, having dual UV and IR interpretations, that are unique to noncommutative theories. These UV-IR divergences, which appear at two loops and beyond, were found to represent dangerous quantum corrections that generally spoil the renormalizability of the theory. Thus, we were led to conjecture that only supersymmetric noncommutative theories that do not receive quantum corrections beyond one-loop are renormalizable, and hence, quantum mechanically consistent.

Furthermore, beyond specific results concerning renormalizability, it is interesting that the consequences of UV-IR mixing in noncommutative quantum theories so profoundly affect the long distance behavior: in $3+1$ dimensions or higher, UV quantum corrections generally introduce new long distance interactions that grow stronger and stronger, even if the gauge coupling is small. In the end, the lesson that we learn is that noncommutative quantum theories exhibit an extreme sensitivity to the UV, such that only the softest UV behavior can be tolerated.

However, there still remain a number of open questions concerning noncommutative quantum field theories. Perhaps, the most glaring omission in our understanding is the nature of quantum effects due to IR states in the theory. This important problem represents an interesting avenue to pursue in future studies. In particular, it would be very satisfying if both the UV and IR dynamics could be encoded together in a unified framework. Surely, this would shed some more light on the fascinating structure of noncommutative theories.

\section{Acknowledgments}

We thank Li Jiang, Sonia Paban, and Willy Fischler for many stimulating discussions. This work was supported by NSF grant PHY-0071512.

\section{APPENDIX A: REVIEW OF THE MATRIX FORMULATION}

For an introduction to the formulation of field theories on noncommutative spaces, see [3, 24], and for a complete review of the matrix formulation of noncommutative gauge theory, see [16, 19]. Here we will review only the main results that we will need for the calculations that appear in this work. Furthermore, we will focus 
only on the treatment of the gauge degrees of freedom, the generalization to matter fields, including fermions, being obvious.

The basic idea of the matrix formulation is that the degrees of freedom are contained in infinite dimensional time dependent hermitian matrices $A_{0}$ and $X^{i}$. In order to separate the quantum effects from UV and IR states, it will prove convenient to work in the background field gauge. In this approach, we expand the fields $A_{0}=B_{0}+$ $A$ and $X^{i}=B^{i}+Y^{i}$ where the background fields, $B_{0}$ and $B^{i}$, are interpreted as the low momentum degrees of freedom, while the fluctuating fields, $A$ and $Y^{i}$, contain the high momentum degrees of freedom. To make the connection with noncommutative gauge theory, we think of $B_{0}=A_{0}(\hat{x})$ and $B^{i}=\hat{x}^{i} \otimes \mathbb{1}_{N \times N}+\theta^{i j} A_{j}(\hat{x})$ where $\hat{x}^{i}$ are infinite dimensional time-independent Hermitian matrices satisfying the algebra of the noncommuting $2 p$ plane

$$
\left[\hat{x}^{i}, \hat{x}^{j}\right]=i \theta^{i j} \mathbb{1}
$$

After a suitable choice of gauge fixing, which includes setting $B_{0}=0$, the Lagrangian takes the form $L=L_{0}+$ $L_{2}+L_{3}+L_{4}$ where

$$
\begin{aligned}
L_{0}= & \operatorname{Tr}\left(\frac{1}{2} \dot{B}^{i 2}+\frac{1}{4}\left[B^{i}, B^{j}\right]\left[B^{i}, B^{j}\right]\right) \\
L_{2}= & \operatorname{Tr}\left(\frac{1}{2} \dot{Y}^{j 2}+\frac{1}{2}\left[B^{i}, Y^{j}\right]^{2}-\frac{1}{2} \dot{A}^{2}-\frac{1}{2}\left[B^{i}, A\right]^{2}+\dot{\bar{c}} \dot{c}\right. \\
& \left.+\left[B^{i}, \bar{c}\right]\left[B^{i}, c\right]+\left[B^{i}, B^{j}\right]\left[Y^{i}, Y^{j}\right]-2 i \dot{B}^{i}\left[A, Y^{i}\right]\right) ; \\
L_{3}= & \operatorname{Tr}\left(\left[B^{i}, A\right]\left[A, Y^{i}\right]+\left[B^{i}, Y^{j}\right]\left[Y^{i}, Y^{j}\right]+\left[B^{i}, \bar{c}\right]\left[Y^{i}, c\right]\right. \\
& \left.-i \dot{Y}^{i}\left[A, Y^{i}\right]-i \dot{\bar{c}}[A, c]\right) ; \\
L_{4}= & \operatorname{Tr}\left(\frac{1}{4}\left[Y^{i}, Y^{j}\right]\left[Y^{i}, Y^{j}\right]-\frac{1}{2}\left[A, Y^{i}\right]^{2}\right) .
\end{aligned}
$$

Notice that we have neglected the linear term in the fluctuating fields, which generically, is not consistent because we expect that UV quantum corrections will contribute to the dynamics of the background field through tadpoles and other effects resulting from the linear interactions. In the language of perturbation theory, this amounts to corrections that are both higher order in the gauge coupling and higher order in derivatives of the background field. Therefore, in order to consistently ignore the linear term, we must require both that the coupling is sufficiently weak so that the loop corrections are suppressed and that the states we integrate out are of sufficiently high momenta relative to the scale set by the background so that the higher derivatives are suppressed, as well. We will find that both of these conditions are met in our approach, so for simplicity sake, we have neglected the linear interactions.

Moving along, from $L_{2}$, we see that up to commutators and time derivatives of the background fields, all of the fluctuating fields, $\Phi=\left(Y^{i}, A, \bar{c}, c\right)$, have similar quadratic terms of the form

$$
\begin{aligned}
& \operatorname{Tr}\left(\frac{1}{2} \dot{\Phi}^{2}+\frac{1}{2}\left[B^{i}, \Phi\right]^{2}\right) \\
& =\frac{1}{2} \Phi^{T}\left(-\mathbb{1} \otimes \mathbb{1} \frac{d^{2}}{d t^{2}}+\left(B^{i} \otimes \mathbb{1}-\mathbb{1} \otimes B^{i}\right)^{2}\right) .
\end{aligned}
$$

For both consistency with the neglect of the linear terms in the fluctuating fields, as discussed above, and convenience in the definition of the propagator for the fluctuating fields, we will treat the commutator and time derivative terms perturbatively while absorbing the remaining background field dependence into the definition of the propagator. As discussed in [16], this choice corresponds to a derivative expansion of the background field, which makes sense because we will only integrate out high momentum states.

To lowest order in the derivative expansion, the propagator for the high momentum modes is

$$
\begin{aligned}
\left\langle\Phi(t) \Phi\left(t^{\prime}\right)^{T}\right\rangle= & \int_{\theta \Lambda} \\
& d^{2 p} x \int_{\Lambda} \frac{d \omega}{2 \pi} \int \frac{d^{2 p} k}{(2 \pi)^{2 p}} e^{-i \omega\left(t-t^{\prime}\right)} \\
& \times e^{i k \cdot\left(x-x^{\prime}\right)} \widetilde{G}\left(\omega, \theta^{-1}\left(x-x^{\prime}\right)\right) \\
& \times e^{-i k \cdot B} \otimes e^{i k \cdot B}
\end{aligned}
$$

where $\widetilde{G}(\omega, p)=\left(\omega^{2}-p_{i} G^{i j} p_{j}\right)^{-1}$ is the momentum space field theory propagator for a massless bosonic state and $G^{i j}$ is the inverse spatial metric, which we will discuss at the end of this section. Were we dealing with fermions instead of bosons, we would simply replace $\widetilde{G}(\omega, p)$ above with the appropriate propagator for fermi fields.

As discussed in [16], Eq. (A4) manifestly describes dipole degrees of freedom whose transverse length is proportional to their center of mass momentum. In particular, the identification is that $x$ and $x^{\prime}$ are the endpoints of the dipole in space, and the center of mass momentum is given by $p=\theta^{-1}\left(x-x^{\prime}\right)$. It is also important to understand that there is a lower cutoff on the integrals over $\omega$ and $x$, which reflects the fact that the Wilsonian integration is only performed over states of high energy and momentum compared to the background.

In practicality, when we perform calculations, we frequently encounter traces of the form 15, 16.

$$
\begin{aligned}
& \operatorname{Tr}\left(\mathcal{O} e^{i k \cdot B}\right) \\
= & \int d^{2 p} x e^{i k \cdot x} \operatorname{tr}_{N}\left(P_{*} e^{i \int_{0}^{1} d \sigma k \cdot \theta \cdot A(x+\sigma k \cdot \theta)} * \mathcal{O}(x)\right) .
\end{aligned}
$$

where $\mathcal{O}$ is some operator in the adjoint representation of the gauge group. We immediately recognize this object as an open Wilson line with $\mathcal{O}(x)$ attached at the end. In fact, this structure was essentially guaranteed by the noncommutative gauge invariance [14, 18]. Furthermore, we will also frequently encounter the Fourier transform of the open Wilson lines above, which we shall denote

$$
\rho_{\mathcal{O}}(x)=\int \frac{d^{2 p} k}{(2 \pi)^{2 p}} e^{i k \cdot x} \operatorname{Tr}\left(\mathcal{O} e^{-i k \cdot B}\right) .
$$


Finally, we should mention that we are using a convention of units in which $(2 \pi)^{2 p} \operatorname{det}(\theta)=1$. This convention could potentially lead to some unfamiliar notions of dimension. What is more, the situation is further complicated by the fact that the inverse spatial metric is $G^{i j}=\theta^{i k} \theta^{k j}$. Nonetheless, the quantities that will appear in our calculations will all have simple physical interpretations. The rule to follow is that the magnitude squared of distance $\left|x_{1}-x_{2}\right|^{2}$ has dimensions of energy squared. The reason is that

$$
\left|x_{12}\right|^{2}=\left(\theta^{-1} \cdot x_{12}\right)_{i} G^{i j}\left(\theta^{-1} \cdot x_{12}\right)_{j}=p_{i} G^{i j} p_{j}
$$

where we have used the relation between the center of mass momentum and the position of the end points of the dipole quanta $p_{i}=\theta_{i j}^{-1}\left(x_{1}^{j}-x_{2}^{j}\right)$. Likewise, by the same argument, the magnitude squared of momenta has dimensions of length squared.

\section{APPENDIX B: LEADING TWO-LOOP CALCULATION}

The leading two-loop contribution to the effective action comes from treating the interaction terms in $L_{4}$ to first order in perturbation theory and the interaction terms in $L_{3}$ to second order. In 16 , the contribution from both the $L_{4}$ terms as well as the time derivative terms in $L_{3}$ was computed. In this section, we will comput the contribution to the effective action from the re- maining terms in $L_{3}$, which can be represented schematically as

$$
\begin{aligned}
\left\langle\int d t_{1} \operatorname{Tr}\right. & \left([\mathrm{B}, \Phi][\Phi, \Phi]\left(\mathrm{t}_{1}\right)\right) \\
& \left.\times \int d t_{2} \operatorname{Tr}\left([\mathrm{B}, \Phi][\Phi, \Phi]\left(\mathrm{t}_{2}\right)\right)\right\rangle
\end{aligned}
$$

where $B$ is the low momentum background field and $\Phi$ represents the high momentum fluctuating fields to be integrated out. The angled brackets denote the vacuum expectation value, which in our background field language, is the product of the expectation value of the low momentum "background fields" and the expectation value of the high momentum "fluctuating fields". Since we are working in the Wilsonian scheme, we evaluate only the latter quantity and think of the residual expression involving an expectation value of background fields as originating from an effective Lagrangian of background fields only.

Furthermore, in the evaluation of Eq. (B1), we neglect the tadpole contractions, although generally, there will be tadpole contributions from the linear interaction term in the Lagrangian as well as both the cubic and quartic interaction terms. However, the linear terms are suppressed by higher derivatives of the background as we have already discussed in Appendix A, and in the supersymmetric theories that are ultimately of interest to us, the tadpoles from interaction terms cancel as well. After a straight forward but somewhat tedious calculation, up to subleading time derivative and commutator terms we have

$$
\begin{aligned}
& \int d t_{1} d t_{2} d^{2 p} x_{1} d^{2 p} x_{2} d^{2 p} x_{3} \int \frac{d \omega_{1} d^{2 p} k_{1}}{2 \pi(2 \pi)^{2 p}} \frac{d \omega_{2} d^{2 p} k_{2}}{2 \pi(2 \pi)^{2 p}} \frac{d \omega_{3} d^{2 p} k_{3}}{2 \pi(2 \pi)^{2 p}} e^{-i \omega_{1}\left(t_{1}-t_{2}\right)+i k_{1} \cdot x_{1}} e^{-i \omega_{2}\left(t_{1}-t_{2}\right)+i k_{2} \cdot x_{2}} e^{-i \omega_{3}\left(t_{1}-t_{2}\right)+i k_{3} \cdot x_{3}} \\
& \times \widetilde{G}\left(\omega_{1}, \theta^{-1} x_{1}\right) \widetilde{G}\left(\omega_{2}, \theta^{-1} x_{2}\right) \widetilde{G}\left(\omega_{3}, \theta^{-1} x_{3}\right) \frac{\partial}{\partial k_{2}} \cdot \frac{\partial}{\partial k_{3}}\left[\operatorname{Tr}\left(\mathrm{e}^{\mathrm{ik} \mathrm{k}_{1} \cdot \mathrm{B}\left(\mathrm{t}_{1}\right)} \mathrm{e}^{\mathrm{i} \mathrm{k}_{2} \cdot \mathrm{B}\left(\mathrm{t}_{1}\right)}\right) \operatorname{Tr}\left(\mathrm{e}^{-\mathrm{ik} \mathrm{k}_{1} \cdot \mathrm{B}\left(\mathrm{t}_{1}\right)} \mathrm{e}^{\mathrm{ik} \mathrm{k}_{3} \cdot \mathrm{B}\left(\mathrm{t}_{1}\right)}\right)\right.
\end{aligned}
$$

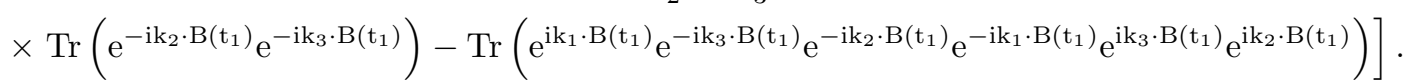

Since we only integrate out states with high energy and momentum, time derivatives and commutators involving the background field are suppressed. Therefore, to lowest order, we obtain

$$
\begin{aligned}
\int d t & d^{2 p} x_{1} d^{2 p} x_{2} d^{2 p} x_{3} \int \frac{d \omega_{1} d^{2 p} k_{1}}{2 \pi(2 \pi)^{2 p}} \frac{d \omega_{2} d^{2 p} k_{2}}{2 \pi(2 \pi)^{2 p}} \frac{d^{2 p} k_{3}}{(2 \pi)^{2 p}} \\
& \times e^{i k_{1} \cdot x_{1}} e^{i k_{2} \cdot x_{2}} e^{i k_{3} \cdot x_{3}} x_{2} \cdot x_{3} \\
& \times \widetilde{G}\left(\omega_{1}, \theta^{-1} x_{1}\right) \widetilde{G}\left(\omega_{2}, \theta^{-1} x_{2}\right) \widetilde{G}\left(\omega_{1}+\omega_{2}, \theta^{-1} x_{3}\right) \\
& \times \operatorname{Tr}\left(\mathrm{e}^{\mathrm{i}\left(\mathrm{k}_{1}+\mathrm{k}_{2}\right) \cdot \mathrm{B}(\mathrm{t})}\right) \operatorname{Tr}\left(\mathrm{e}^{-\mathrm{i}\left(\mathrm{k}_{1}-\mathrm{k}_{3}\right) \cdot \mathrm{B}(\mathrm{t})}\right) \\
& \times \operatorname{Tr}\left(\mathrm{e}^{-\mathrm{i}\left(\mathrm{k}_{2}+\mathrm{k}_{3}\right) \cdot \mathrm{B}(\mathrm{t})}\right)
\end{aligned}
$$

after performing the integral over $\omega_{3}$ and $t_{2}$ and integrating by parts in the $k$ integrals. Note that we have dropped the single trace term, since as discussed in [16], it corresponds to a nonplanar matrix diagram, which is suppressed in the domain of Wilsonian integration. Finally, upon Fourier transforming back to position space, we are left with

$$
\begin{aligned}
\int d t & d^{2 p} x_{1} d^{2 p} x_{2} d^{2 p} x_{3} \int \frac{d \omega_{1}}{2 \pi} \frac{d \omega_{2}}{2 \pi} x_{23} \cdot x_{31} \\
& \times \widetilde{G}\left(\omega_{1}, \theta^{-1} x_{12}\right) \widetilde{G}\left(\omega_{2}, \theta^{-1} x_{23}\right) \widetilde{G}\left(\omega_{1}+\omega_{2}, \theta^{-1} x_{31}\right) \\
& \times \rho\left(x_{1}, t\right) \rho\left(x_{2}, t\right) \rho\left(x_{3}, t\right) .
\end{aligned}
$$

We can now combine the above expression with the 
result that was calculated in [16] to obtain the leading two-loop contribution from the second order treatment of all of the cubic interactions in $L_{3}$. We get a result proportional to

$$
\begin{aligned}
\int d t & d^{2 p} x_{1} d^{2 p} x_{2} d^{2 p} x_{3} \int \frac{d \omega_{1}}{2 \pi} \frac{d \omega_{2}}{2 \pi}\left(\omega_{1} \omega_{2}-x_{12} \cdot x_{23}\right) \\
& \times \widetilde{G}\left(\omega_{1}, \theta^{-1} x_{12}\right) \widetilde{G}\left(\omega_{2}, \theta^{-1} x_{23}\right) \widetilde{G}\left(\omega_{1}+\omega_{2}, \theta^{-1} x_{31}\right) \\
& \times \rho\left(x_{1}, t\right) \rho\left(x_{2}, t\right) \rho\left(x_{3}, t\right) .
\end{aligned}
$$

Note that we have used the $S O(1,2 p)$ symmetry that is present in the vacuum diagrams in order to determine that the integrand of the combined expression can only depend on the invariant product $\omega_{1} \omega_{2}-x_{12} \cdot x_{23}$. With this factor present in the integrand, as in the commutative theories, the two-loop cubic interactions given by Eq. (B5) can be reduced to the form of Eq. (8).

\section{APPENDIX C: NEXT TO LEADING ORDER TWO LOOP CALCULATION}

As in the next to leading order one-loop calculation, to get the precise result we must retain higher order commutators and time derivatives that were dropped in the derivation of the matrix propagator (A4) as well as the field strength terms in $L_{2}$ that were also excluded from the the propagator 16. However, after developing some intuition in section II for the structure of terms that can appear, a lengthly calculation is not necessary. We simply need to keep track of all the distinct possibilities in which the field strength insertions can appear in the diagrams. For example, in the case of the quartic diagram, both insertions can go into one loop or each loop can get a single insertion

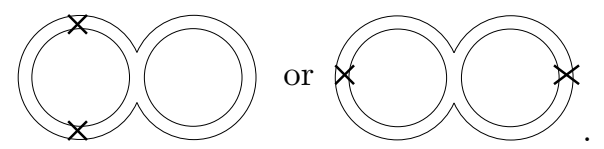

Of course, each insertion requires an extra field theory propagator to appear in the corresponding loop, and for each loop, there are two boundaries in which the insertion can go. Therefore, the first diagram corresponding to both insertions going into the same loop contributes two terms

$$
\begin{aligned}
\int d^{2 p} & x_{1} d^{2 p} x_{2} d^{2 p} x_{3} \int d \omega_{1} d \omega_{2} \\
& \times \widetilde{G}\left(\omega_{1}, \theta^{-1} x_{13}\right)^{3} \widetilde{G}\left(\omega_{2}, \theta^{-1} x_{23}\right) \\
& \times\left[b_{1} \rho_{F F}\left(x_{1}\right) \rho\left(x_{2}\right) \rho\left(x_{3}\right)+b_{2} \rho_{F}\left(x_{1}\right) \rho\left(x_{2}\right) \rho_{F}\left(x_{3}\right)\right],
\end{aligned}
$$

[1] N. Seiberg and E. Witten, J. High Energy Phys. 09, 032 (1999).

[2] M. M. Sheikh-Jabbari, Phys. Lett. B 455, 129 (1999); while the second diagram corresponding to one insertion going into each loop contributes three terms

$$
\begin{aligned}
\int d^{2 p} & x_{1} d^{2 p} x_{2} d^{2 p} x_{3} \int d \omega_{1} d \omega_{2} \\
& \times \widetilde{G}\left(\omega_{1}, \theta^{-1} x_{13}\right)^{2} \widetilde{G}\left(\omega_{2}, \theta^{-1} x_{23}\right)^{2} \\
\times & \quad\left(b_{3} \rho_{F}\left(x_{1}\right) \rho\left(x_{2}\right)_{F} \rho\left(x_{3}\right)+b_{4} \rho\left(x_{1}\right) \rho\left(x_{2}\right) \rho_{F F}\left(x_{3}\right)\right. \\
& \left.+b_{5} \rho_{F}\left(x_{1}\right) \rho\left(x_{2}\right) \rho_{F}\left(x_{3}\right)\right] .
\end{aligned}
$$

There are many more possibilities in the case of the cubic graph. For simplicity, we will enumerate them in four propagator form, which is obtained after the cancellation of one propagator by the momenta in the numerator of the integrand (see Eq. (B5) ). It is not difficult to see that there are three combinations of field theory propagators that emerge: the first is similar to Eq. (C1), the second is similar to Eq. (C2), and the third is given by

$$
\begin{aligned}
\int d^{2 p} & x_{1} d^{2 p} x_{2} d^{2 p} x_{3} \int d \omega_{1} d \omega_{2} \\
& \times \widetilde{G}\left(\omega_{1}, \theta^{-1} x_{12}\right)^{2} \widetilde{G}\left(\omega_{2}, \theta^{-1} x_{23}\right) \widetilde{G}\left(\omega_{1}+\omega_{2}, \theta^{-1} x_{13}\right) \\
& \times\left[b_{6} \rho_{F F}\left(x_{1}\right) \rho\left(x_{2}\right) \rho\left(x_{3}\right)+b_{7} \rho_{F}\left(x_{1}\right) \rho_{F}\left(x_{2}\right) \rho\left(x_{3}\right)\right. \\
& \left.+b_{8} \rho_{F}\left(x_{1}\right) \rho\left(x_{2}\right) \rho_{F}\left(x_{3}\right)\right] .
\end{aligned}
$$

Let us now discuss the meaning of these expressions in the context of supersymmetric theories. First of all, consider the terms of the form Eq. (C1). The planar subdiagram corresponding to the constant term of $\rho\left(x_{2}\right)$, contributes one-loop divergent mass corrections to the propagators appearing in Eq. (7). As discussed in section III, in supersymmetric theories, contributions of this type will always cancel.

Another obvious group is the combination given by Eq. (C2). By isolating the constant term of $\rho\left(x_{2}\right)$, we recover the UV divergent one-loop correction to the gauge coupling that multiplies Eq. (7). Of course, these types of corrections are required by renormalizability and pose no threat to the consistency of the theory. Moreover, the constant term of $\rho\left(x_{3}\right)$ gives rise to a benign quantum correction to the $\rho_{F} \rho_{F}$ interaction in Eq. (7).

Finally, we arrive at the dangerous group of terms given by Eq. (C3). Although, the constant term of $\rho\left(x_{2}\right)$ gives a harmless quantum correction to the interaction strength of Eq. (7), the constant term of $\rho\left(x_{3}\right)$ leads to the same type of dangerous UV-IR divergent correction that was discussed at length in section III. Therefore, renormalizability demands that these contributions vanish.
D. Bigatti and L. Susskind, Phys. Rev. D 62, 066004 (2000); Z. Yin, Phys. Lett. B 466, 234 (1999); H. Liu and J. Michelson, Phys. Rev. D 62, 066003 (2000). 
[3] S. Minwalla, M. Van Raamsdonk and N. Seiberg, J. High Energy Phys. 02, 020 (2000).

[4] C.P. Martin and D. Sanchez-Ruiz, Phys. Rev. Lett. 83, 476 (1999); T. Krajewski and R. Wulkenhaar, Int. J. Mod. Phys. A 15, 1011 (2000).

[5] C.P. Martin and F.R. Ruiz, Nucl. Phys. B597, 197 (2001).

[6] I. Chepelev and R. Roiban, J. High Energy Phys. 05, 037 (2000); H. Grosse, T. Krajewski, and R. Wulkenhaar ; H. Girotti, M. Gomes, V. Rivelles, and A. da Silva, Nucl. Phys. B587, 299 (2000); I. Chepelev and R. Roiban, J. High Energy Phys. 03, 001 (2001); A. Bichl, et al, J. High Energy Phys. 06, 013 (2001); S. Sarkar, J. High Energy Phys. 06, 003 (2002).

[7] M. Hayakawa, Phys. Lett. B 478, 394 (2000).

[8] F.R. Ruiz, Phys. Lett. B 502, 274 (2001).

[9] V. V. Khoze and G. Travaglini, J. High Energy Phys. 01, 026 (2001).

[10] L. Griguolo and M. Pietroni, J. High Energy Phys. 05, 032 (2001).

[11] G.H. Chen and Y.S. Yu, Nucl. Phys. B622, 189 (2002).

[12] Y. Kiem, S. Lee, S. J. Rey and H. T. Sato, hepth/0110215; Y. Kiem, S. J. Rey, H. T. Sato and J. T. Yee, Phys. Rev. D 65, 026002 (2002); hep-th/0107106; Y. Kiem, S. S. Kim, S. J. Rey and H. T. Sato, hepth/0110066.
[13] A. Armoni and E. Lopez, Nucl. Phys. B632, 240 (2002).

[14] H. Liu and J. Michelson, Nucl. Phys. B614, 279 (2001); H. Liu, ibid. B614, 305 (2001).

[15] M. Van Raamsdonk, J. High Energy Phys. 11, 006 (2001).

[16] L. Jiang and E. Nicholson, Phys. Rev. D 65, 105020 (2002).

[17] S. Iso, H. Kawai, and Y. Kitazawa, Nucl. Phys. B576, 375 (2000).

[18] N. Ishibashi, S. Iso, H. Kawai and Y. Kitazawa, Nucl. Phys. B573, 573 (2000); S. J. Rey and R. von Unge, Phys. Lett. B 499, 215 (2001); S. Das and S. J. Rey, Nucl. Phys. B590, 453 (2000); D. Gross, A. Hashimoto and N. Itzhaki, Adv. Theor. Math. Phys. 4, 893 (2000).

[19] N. Seiberg, J. High Energy Phys. 09, 003 (2000).

[20] A. Matusis, L. Susskind and N. Toumbas, J. High Energy Phys. 12, 002 (2000).

[21] D. Zanon, Phys. Lett. B 502, 265 (2001).

[22] R. Gopakumar, S. Minwalla, N. Seiberg, and A. Strominger, J. High Energy Phys. 08, 008 (2000).

[23] A. Rajaraman and M. Rozali, J. High Energy Phys. 04, 033 (2000).

[24] R. Gopakumar, S. Minwalla and A. Strominger, J. High Energy Phys. 05, 020 (2000). 